\title{
Enabling New Science Mission Capabilities with Additive Manufacturing Technologies
}

\author{
Dr. Bryan McEnerney bryan.mcenerney@jpl.nasa.gov and \\ Dr. R. Peter Dillon robert.p.dillon@jpl.nasa.gov \\ (Jet Propulsion Laboratory, California Institute of Technology) \\ with \\ John Vickers (NASA MSFC) \\ Karen Taminger (NASA LaRC) \\ Dr. Steve Jolly (Lockheed Martin Space) \\ Professor Tony Rollett (Carnegie Mellon University) \\ endorsed by \\ Majid Babai, Advanced Manufacturing Chief, (NASA Marshall Space Flight Center) \\ Dr. Patricia M. Beauchamp (Jet Propulsion Lab, California Institute of Technology) \\ Dr. Anthony Freeman (Jet Propulsion Laboratory, California Institute of Technology) \\ Tim O'Donnell (Jet Propulsion Laboratory, California Institute of Technology) \\ Kim R. Reh (Jet Propulsion Laboratory, California Institute of Technology)
}

\section{A White Paper Submitted to the Decadal Survey on Planetary Science and Astrobiology 2023-2032}

September 15, 2020

Cost, mass, volume and schedule limit the extent of science and discovery that can be integrated into many Planetary Science and Astrobiology Missions. Manufacturing, materials and structures are key contributors to these limiting factors, and additive manufacturing (AM) offers integrated solutions to these challenges. Despite worldwide investment in AM, NASA is uniquely positioned to address the singular challenges of Planetary Science and Astrobiology Missions. Specific research and development needs require immediate attention and investment to capitalize on the benefits $A M$ offers.

\section{NASA Has a Strong Background in Additive Manufacturing Development}

Additive manufacturing (AM), also known as 3D printing, is a transformative technology that vastly broadens the design space for optimizing materials, parts, and systems for space applications. AM can deliver tremendous performance improvements by enabling more complex and multi-capable material and structure combinations than previously possible. The potential to significant reduce development and fabrication cycle time can provide substantial budget and schedule flexibility for complex missions. A major NASA priority within the science community should be the development of advanced AM solutions targeting future Planetary Science and Astrobiology Missions.

NASA has a strong history in AM activities that support the full range of NASA's missions and span all NASA centers. NASA's expertise in AM has benefitted the aerospace industry, other Government Agencies, and addressed critical national needs. NASA performs applied research and development (R\&D) at NASA centers, through 
contracts/grants, and in public-private partnerships to focus on NASA-specific mission capabilities such as propulsion, in-space manufacturing, science and aeronautics. The agency leverages general AM developments made by other government agencies (e.g. DOD and DOE), universities and commercial partnerships.

AM enables novel architectural designs such as multi-functional elements and performance-optimized systems that can meet tight spacecraft budgets for mass, volume, and power. Cross-institution partnering will foster and accelerate fundamental breakthroughs in materials and design/analysis paradigms, leading to new approaches for scientific exploration. The integration of advanced materials development, component design and analysis, and manufacturing through AM is game-changing in its impact on future Planetary and Astrobiology missions-influencing engineering practice and improving scientific return. The development of this document involved the participation of national-level AM Technologists as well as NASA mission programmatic leadership.

\section{Early Adoption of AM in NASA Missions}

There is precedence for using AM to build unique components for NASA Science Missions. Some of the first AM parts ever to be flown on a NASA Science Mission were developed by Lockheed for the JPL Juno Mission in the 2011 timeframe. NASA's flagship Mars 2020 mission is the first major science mission where AM is an enabling technology for two payloads: the Planetary Instrument for X-ray Lithochemistry (PIXL) science instrument and the Mars Oxygen In-Situ Resource Utilization Experiment (MOXIE). AM was an enabling technology for PIXL's structure/pointing system, offering mass savings of well over $50 \%$, in addition to creating structural features that were fabricated as a single-piece, but which would have required $2-3$ pieces in a conventional design. A total of six parts were fabricated using powder bed fusion technologies, including primary structural mounts and dust covers, Figure 1. MOXIE's high temperature heat exchanger with fine-scale gas vias was developed and implemented faster than a conventional brazed or welded geometry and further offered cost savings versus the conventional technology. These technologies were only feasible due to significant NASA/JPL investment into the fundamental process/property relationships for the respective alloy systems, advanced materials testing, and qualification methodologies, and involvement with national materials property development efforts.
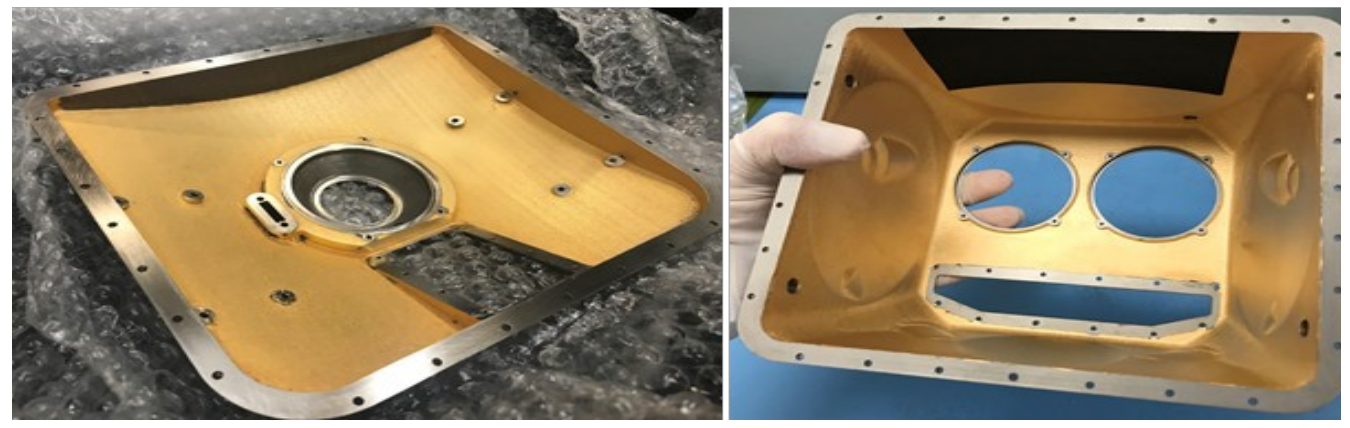

Figure 1 - M2020 PIXL instrument top and bottom Ti-6Al-4V AM covers used to hermetically seal the instrument from contact with Martian rocks. 


\section{The Case for Expanded Implementation of AM for Future Science Missions}

The amount of R\&D focused on fundamental scientific and engineering challenges in AM has been tremendous as it is widely seen as being a transformative technology. However, NASA has not specifically leveraged the lower technology readiness level (TRL) investments into cogent development efforts with a focused application for planetary/astrobiology missions. According to The President's Council of Advisors on Science and Technology (PCAST), in their report entitled 'Recommendations for Strengthening American Leadership in Industries of the Future' dated June 2020, "Industries of the Future (IotF) Institutes should facilitate the development of digital twins and help to expand the capabilities of the domestic manufacturing supply chain as a pathway to Factories of the Future. PCAST envisions Factories of the Future (FotF) leveraging advanced physical and virtual assets for dramatically enhanced versatility and efficiency. lotF Institutes will serve as a mechanism for conceiving, testing, and ultimately implementing these benefits. For example, additive manufacturing technologies such as 3D printing will provide on-demand adaptation and customization of parts, enabling on-the-spot repairs and improvements in manufacturing operations." Additionally, the White House published memorandum outlining the FY22 R\&D priorities for federal agencies states "departments and agencies should seek opportunities to work with advanced materials, additive manufacturing, and machine learning capabilities that have broad potential applications in space and on Earth."

NASA has already laid the groundwork to enable widespread adoption and implementation of AM into future NASA Science Missions. Examples of on-going or recent $A M-$ related $R \& D$ include:

- Developed at NASA LaRC, In-situ Characterization and Inspection of Additive Manufacturing Deposits using Transient Infrared Thermography may provide a superior in situ, non-destructive inspection technique. The method provides transient temperature profiles of the surface, including the melt pool, at each step/layer. This system can measure material properties and detect internal and surface defects, allowing for characterization of the deposition quality in real-time. The quantitative inspection images, is a more effective way of determining flaws and deposition quality during the build process and can be used to document the structural integrity during ground-based and space-based deposition.

- NASA JPL has provided key content in materials modeling, design, and analysis with the development of the advanced physics based software toolset, pycalphad. Utilizing the CALPHAD (Computer Coupling of Phase Diagrams and Thermochemistry) method this toolset enables design of new materials. Using computational thermodynamics to predict phase formation and identify potential build paths for multicomponent systems, the properties can be spatially tailored to meet design requirements.

- NASA GSFC is developing next generation AM for multifunctional sensors made with nanomaterials that will benefit a broad range of missions across Planetary Science, Earth Science, Heliophysics, and Human Exploration.

- NASA MSFC has recently 3D printed bi-metallic copper-based alloys GRCop-84/42, for use in rocket propulsion components, and continues to move the bar for AM of propulsion technology with the support of other NASA centers through the Rapid Analysis and Manufacturing Propulsion Technology (RAMPT) project. 
- Wire-feed Electron Beam AM (Wire-EBAM) has been successfully used in the aerospace industry for the production of large pressure vessels, the kinds typically used on all NASA Science Missions. Lockheed Martin used Wire-EBAM to produce titanium fuel tanks for satellites, resulting in significant reduction in time and cost.

- Multiple NASA R\&D applications are inspired by industry and corporate development of metal powder, the feedstock for blown powder and powder bed AM. More than one NASA organization has recently procured new high strength aluminum alloy powders, developed specifically for AM processing, that offer the potential to produce structural parts via AM with mechanical properties comparable to their wrought cousins, the 6061,7050 , and 7075 aluminum alloys. Mainstream adoption by NASA flight project engineers is anticipated with sufficient funding to develop and qualify the first few introductory parts fabricated by AM using these advanced materials.

- Hiperco ${ }^{\circledR}$, an iron-cobalt alloy, has been recently additively manufactured and thermally treated to generate favorable soft ferromagnetic properties by NASA JPL and DOE's Sandia Labs for use in advanced applications such as magneticallyshielded Hall thrusters, high-efficiency motors, and low-loss power systems.

- Project Gamma (Autodesk and NASA JPL) produced generatively-designed Europa Lander prototype hardware (Figure 2) with the potential for $30 \%$ mass saving compared to the baseline design. This is achievable with a combination of AM fabricated tooling for cast components and components directly fabricated by AM.

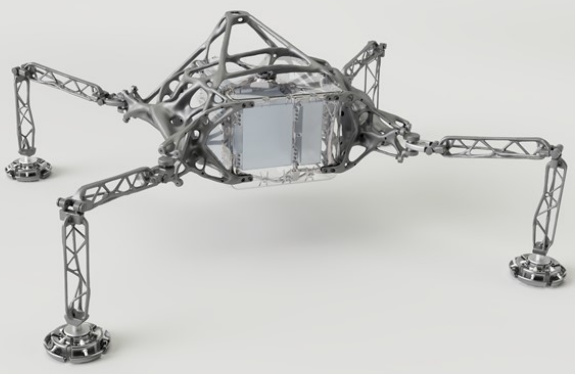

Figure 2 - Project Gamma's Europa Lander design concept. Pre-Decisional Information -- For Planning and Discussion Purposes Only

\section{Future AM R\&D Needs for Planetary Science and Astrobiology Missions}

A key to this effort will be identifying specific NASA targets of opportunity especially within the context of upcoming mission architectures. Some examples of AM areas that should be prioritized include:

Complex multi-functional geometries that are otherwise difficult or impossible to manufacture; integrated propulsion components and/or thermal management within structure are two examples. These types of structures can act as primary, secondary, or non-loaded structures while also providing additional mechanical, thermal, propulsive, electromagnetic, electrical, or actuation functionalities. Multi-functional space components have been envisioned for decades as a means to reduce mass and size, but AM finally provides a means to build them.

Embedded sensors and integrated actuators can be 3D printed with AM for integrated component/system health assessment critical for onboard autonomy or mechanism-free joints printed with shaped memory alloys. 
Compositional or microstructural gradients allows property variation within a structure that is not easily achievable without AM. This technique can be used to spatially tailor structural, thermal, optomechanical, electrical, RF, or other properties for optimized instrument/spacecraft performance. A specific example of this approach would be functionally grading a material to avoid magnetic response in an area of the component adjacent to magnetically sensitive equipment (e.g. magnetometers).

Bulk metallic glass (BMG) alloys have extremely high wear resistance and low friction coefficients for gears and actuators. The rapid cooling rates in AM processes avoid crystallization of BMG alloys enable fabrication of amorphous components much larger than the critical casting thickness of the alloy. With additional investment, the current BMG gear technology NASA has developed to enable heaterless gearboxes that function at $100^{\circ} \mathrm{K}$ could be scaled larger thus increasing the design space for BMGbased actuators. This could be enabling for missions to Ocean Worlds such as Europa and Enceladus, as well as Lunar Missions, where power is a precious commodity.

High performance, tailored materials and complex shapes are traditionally difficult to process, but are attainable with AM. More fundamental R\&D involving materials tailoring and development of high-entropy alloys (HEA) systems such as multi-principal element alloys (MPEA) for structural and functional materials, and complex concentrated alloys (CCA) with metallic, ionic, covalent bonding or many different material phases can provide future breakthroughs for NASA Science Missions. A very compelling motivation is AM-produced complex shapes from ceramics, functionally-graded metal alloys, highperformance aluminum alloys, smart materials, RF materials, electromagnetic materials, carbon fiber reinforced polymers, electronic printed wiring boards, or printable polyimide (Kapton) uniquely targeted for future NASA applications.

\section{NASA Has a Specific Role to Play in AM Development for Science Missions}

The majority of the work performed in academia is focused on low-TRL research without a clear transition path to a viable application for NASA. Concurrently, most of the industrial development is happening outside of the aerospace industry, and while more applied and higher TRL, does not focus on space-related applications or materials. The work that is relevant to NASA is often focused on crewed spaceflight or low Earth orbit (LEO) applications (e.g. Inconel 718 for liquid rocket engine applications). Consequently, nearly all fundamental and most applied research in materials will require tailoring for deep space applications, especially for the harsher conditions in Icy Worlds and Lunar missions. Therefore, it is important for NASA to establish a range of partnerships with academia and industry and to leverage and focus advances for use on future planetary/astrobiology missions. Further, it would be highly advisable for NASA to begin understanding the limitations of current spacecraft architecture to generate a series of design approaches, software, and hardware tools to meet the most challenging mission requirements.

Topology Optimization: One such design approach is Topology Optimization (TO), a mathematical framework used to computationally design a component for optimal performance (e.g. structural, thermal, optical and combinations thereof) based on relevant constraints and loading conditions. The resulting designs, such as lattice structures for mesh wheels or impact structures, are challenging to build with conventional manufacturing, but attainable via AM. The most common application of TO 
is structural optimization which can result in tremendous mass savings; however, greater benefits may be reaped by optimizing for unique combinations of mission environmental conditions and technical performance specifications. With appropriate future funding, tools and analysis techniques to handle multi-performance, NASAunique constraints will continue to offer increased capability to deliver functionally and mass-optimized systems.

AM Design Tools: Attendant with AM developments for NASA Mission applications is the need for significantly increased design tool capability. There has already been a large investment in tool development within both the commercial and government sectors. For example, Airbus has flown topologically optimized parts for the Eurostar E3000 satellite, as has ESA for its Sentinal-1 satellite constellation. The focused areas of priority for targeted NASA development, likely with partners in academia, are in the areas of AM design tools, including multi-physics modeling and simulation (e.g. heat transfer, telecommunication/ scientific applications, optical, electronics) and tools for concurrent and non-linear design optimization. Other targeted aerospace tool capabilities (e.g. consideration for buckling and manufacturing constraints) should be developed through partnerships with other government agencies and industry.

More Capable CAD/Analysis Tools: The greatly expanded scope of AM design space, addressing non-linear optimization problems along with the opportunity to design and build much more intricate geometries, are stretching current computer aided design (CAD) tools to their limit. Lattice design is an especially apt example, where novel designs can greatly tax most current CAD software packages. Furthermore, current feature-based CAD is not well-suited for the challenges of AM design, and those tools will need to be expanded to handle complex geometries and computationally intensive analysis and optimization. For NASA, this translates into the need of transitioning its design and analysis tools onto higher-performance platforms, requiring tool restructuring such as parallelization to allow simultaneous processing on multiple systems.

Hybrid Manufacturing: Hybrid manufacturing refers to employing combinations of multiple production methodologies to create a single part, such that all elements of the produced part can be optimally controlled. Combining Computed Numerical Control machining with AM will produce precision, complex geometries and surface finishes not attainable by AM alone. Similarly, multi-scale printing, the ability to print structures from meter scale to nanometer scale, all within a single part, is a necessary technology development to create macro-scale structures that have nano- to micro-scale devices integrated into them. Manufacturing techniques for nano- to micro-scale parts exist for electronic, electro-optical, and mechanical devices, as well as for their integration into miniature micro-electromechanical structures (MEMS). In collaboration with academia and research institutes, NASA should be assessing and investing in such technologyrich opportunities for miniature flight and instrument systems.

Verification \& Validation: The transition to employing new AM methods and materials has and will continue to introduce associated technology development needs in the areas of quality assurance, verification and validation $(V \& V)$. Since the fabrication methodology and the associated performance vulnerabilities will be different from traditionally-manufactured components, NASA will need to help develop with industry new testing and qualification methodologies. Additionally, NASA will also need to further its understanding of uncertainty quantification/performance sensitivity for AM process 
control. The key new technology development here is quantification of variation in process and its effect on performance: in principle, once the sensitivity is quantified, the performance risk can be mitigated to a desired tolerance, using for example, Bayesian inference. This is a challenging multiscale measurement and modeling problem, requiring a deep understanding of the underlying process, material, and machine.

Tool Integration: A trend that NASA will need to embrace through new training and pathfinder project efforts over the next 10 years is the continued merging of what is now a relatively compartmentalized discipline-based development in AM. Materials modeling tools, design tools, multi-physics-based analysis tools, and AM manufacturing tools are highly interrelated which will lead to integrated solutions. NASA will need to move from today's mainly serial hardware development processes, consisting of islands of locally optimized components and subsystems, to an integrated, system-level optimization approach capable of achieving the truly advanced space product developments required for planetary science and astrobiology missions in the 2023 - 2032 timeframe.

\section{Summary and Call to Action}

The full implementation of $\mathrm{AM}$ and associated design, materials, analysis, and quality assurance within NASA has the potential to positively transform how most spacecraft and instrument hardware is developed and qualified for flight. AM is not just another new manufacturing technology to be added to the existing portfolio of options: AM enables new material composition options, more complex design space implementation, mass savings, vastly reduced part counts with concomitant simplification of analysis and qualification processes as well as reduced development costs and schedules. AM development is entwined with necessary advancement in key planetary science and astrobiology strategic technology areas such miniaturized systems, distributed systems, communication and navigation, instruments and sensors, as well as robotics and mobility systems. AM can enable mission architecture concepts not presently possible, such as a robust SmallSat Science mission platform with electronics, thermal control, harnessing, propulsion elements, and radiation shielding all incorporated into its structure while still hosting a scientifically capable payload. Advanced architectures dependent upon advances in AM will be particularly important for challenging future missions such as those to Europa, Enceladus, Venus, Titan, and Ceres and outward looking astrobiology missions such as Habex and Luvoir.

Fully engaging in AM technology will allow NASA and associated industry to evolve over time from existing design, analysis, review, and V\&V systems to more efficient approaches, requiring fewer resources throughout the design, fabrication, and qualification process. It will enable not only better part designs in existing systems, but wholly new system design approaches. Multi-material prints, embedded electronics and sensors, and large-scale multi-functional structures are all realizable on the 10-year horizon. In the far future, full seamless integration with advanced, smart computer-aided design, analysis tools, printer technologies, part verification techniques, and test processes will allow ultra-short turnarounds of engineering designs, revolutionizing space system design, cost, and schedule. 
This work was carried out at the Jet Propulsion Laboratory, California Institute of Technology, under a contract with the National Aeronautics and Space Administration (80NM0018D0004). @ 2020. All rights reserved. 